A SYSTEMATIC REVIEW OF THE RISK OF MOTOR VEHICLE COLLISION AND DEMENTIA

D. Carr ${ }^{1}$, M. Rapoport, $\mathrm{MD}^{2}, 1$. Washington University, St Louis, St Louis, Minnesota, United States, 2. University of Toronto, Toronto, Canada

Guidelines that physicians use to assess fitness to drive for dementia are limited in their currency, applicability, and rigor of development. We performed a systematic review to determine the risk of motor vehicle collisions or driving impairment caused by dementia. Seven literature databases retrieved 12,860 search results: we included nine studies in this analysis, involving 378 participants with dementia and 416 healthy controls. We found medium to large effects of dementia on driving abilities in six of the seven recent studies that examined driving impairment. Persons with dementia were more likely to fail a road test than healthy controls (RR: $10.77,95 \% \mathrm{CI}$ : $3.00-38.62, \mathrm{z}=3.65, \mathrm{p}<0.001)$, with no significant heterogeneity $(\chi 2=1.50, \mathrm{p}=0.68, \mathrm{I} 2=0 \%)$. Even mild stages of dementia place patients at substantially higher risk of failing a performance-based road test and of demonstrating impaired driving abilities on the road.

\section{SYSTEMATIC REVIEW OF THE RISK OF MOTOR VEHICLE COLLISION AFTER STROKE OR TRANSIENT ISCHEMIC ATTACK}

R. Marottoli ${ }^{1}$, M. Rapoport, $\mathrm{MD}^{2}$, M. Bayley, $\mathrm{MD}^{3}$, H.M. Finestone, $\mathrm{MD}^{4}$, 1. Yale School of Medicine, New Haven, Connecticut, United States, 2. University of Toronto, Toronto, Canada, 3. University of Toronto, Toronto, Canada, 4. University of Ottawa, Ottawa, Canada

Stroke can impair the motor and cognitive skills required to drive safely. The purpose of the present study was to determine whether stroke and/or transient ischemic attack (TIA) are associated with an increased MVC risk. We searched MEDLINE, CINAHL, EMBASE, PsychINFO and TRID through December 2016. Results: From the 5,605 citations identified, 12 articles met our inclusion criteria. Only one of three case-control studies showed an increased risk of stroke in persons with at-fault MVC (OR 1.9, 95\% CI 1.0-3.9). Of five cohort reports, only one study, limited to self-report, found an increased risk of MVC associated with stroke or TIA (RR 2.71, 95\% CI 1.11-6.61). Discussion: The decline in driving skills following stroke identified in prior reviews does not appear to translate into a robust increase in risk of MVCs. However, there is a paucity of high quality studies of the impact of stroke on driving.

\section{SESSION 2105 (SYMPOSIUM)}

\section{NIA SUPPORT FOR UNDERSTANDING OF THE PURPOSES OF LONGER LIVES-SYMPOSIUM FOR ESTABLISHED RESEARCHERS}

Chair: M.A. Bernard, NIA NIH, Bethesda, Maryland Co-Chair: R. Hodes, National Institute on Aging, National Institutes of Health, Bethesda, Maryland

The National Institute on Aging (NIA) at the National Institutes of Health, Department of Health and Human Services, is the federally designated lead agency on aging research, and has supported significant research on facilitating the aging process, whether from the perspective of the basic biology of aging, the clinical ramifications of the aging process, or behavioral and social aspects of the aging process. NIA is also the federally designated lead agency for Alzheimer's research, with the NIA budget nearly doubling in the last few years, largely but not exclusively due to increased Congressional allocations targeted at Alzheimer's disease. This symposium will provide a forum for established researchers to explore the implications of the recent budget increases for the general research community. It will involve NIA's senior staff discussing research priorities and programs supported by the Institute. A question-and-answer session will follow brief introductory remarks on current funding and future priorities and research directions of NIA. Learning objectives: 1) describe the current and future research foci of the NIA extramural divisions supporting basic, clinical, neuroscience, behavioral and social research; 2) discuss the current funding environment; and 3) provide a forum for discussion among the research community and NIA leadership.

\section{HIGHLIGHTS OF NEUROSCIENCE RESEARCH}

E. Masliah, National Institute on Aging, National Institutes of Health, Bethesda, Maryland, United States

Dr. Masliah will briefly highlight opportunities for research supported by the Division of Neurosciences, including Alzheimer's and non-Alzheimer's research.

\section{HIGHLIGHTS OF BEHAVIORAL AND SOCIAL SCIENCE RESEARCH}

J. Haaga, National Institute on Aging, National Institutes of Health, Bethesda, Maryland, United States

Dr. Haaga will briefly highlight opportunities for research in the behavioral and social sciences supported by his division. This will include Alzheimer's relevant as well as nonAlzheimer's research.

\section{HIGHLIGHTS OF AGING BIOLOGY RESEARCH}

F. Sierra, National Institute on Aging, National Institutes of Health, Bethesda, Maryland, United States

Dr. Sierra will briefly share highlights of research supported by the Division of Aging Biology, with particular reference to geroscience.

\section{HIGHLIGHT OF GERIATRICS AND CLINICAL GERONTOLOGY RESEARCH}

E. Hadley, National Institute on Aging, National Institutes of Health, Bethesda, Maryland, United States

Dr. Hadley will briefly share highlights of research supported and opportunities within the Division of Geriatrics and Clinical Gerontology.

\section{HIGHLIGHTS FROM THE DIVISION OF EXTRAMURAL RESEARCH}

R. Barr, National Institute on Aging, National Institutes of Health, Bethesda, Maryland, United States

Dr. Barr will briefly share perspectives regarding grants funding opportunities and training, managed by the Division of Extramural Activities. 